\title{
Exercise-based cardiac rehabilitation in cardiac resynchronization therapy recipients: A primer for practicing clinicians
}

\author{
Marco Ambrosetti1, Simona Sarzi Braga2, Franco Giada33, Roberto F.E. Pedretti² \\ 1 Cardiovascular Rehabilitation Unit, Le Terrazze Clinic, Cunardo (VA) \\ 2 Department of Cardiac rehabilitation, Istituti Clinici Scientifici Maugeri, IRCCS Tradate, Tradate (VA) \\ 3 Cardiovascular Rehabilitation and Sports Medicine Service, Cardiovascular Department, PF Calvi Hospital, \\ Noale (VE), Italy
}

\begin{abstract}
Cardiac resynchronization therapy (CRT) is a therapeutic option of increasing importance for chronic heart failure (CHF) and criteria for implantation now concern a large amount of patient populations. As a consequence, subjects with ongoing CRT (or immediately after CRT implantation) are more often referred to Cardiac Rehabilitation (CR) programmes, and it has been recently estimated that about one third of CHF patients attending CR in Italy currently have this kind of device. The presence of CRT represents a modulating factor for exercise prescription and monitoring, since CRT patients may be considered per se as a target group for CR. Exercise therapy (ET) increases benefits from CRT on functional capacity, and recent evidence suggests an adjuvant role of ET in improving cardiovascular prognosis also. Both aerobic endurance and resistance training activities may involve CHF patients with CRT, while the potential role of aerobic interval training needs more studies and evidence. Prescription of an ET program should be associated with information regarding device programming and possible limiting factors associated with pacing therapy, tailoring of the basic principles of ET (in terms of type of exercise, intensity and program duration) in this patient group is mandatory.
\end{abstract}

Corresponding author: Marco Ambrosetti, Cardiovascular Rehabilitation Unit, Le Terrazze Clinic, Via U. Foscolo 6/b, 21035 Cunardo (VA), Italy. Tel.+39.0332.992448. E-mail: m.ambrosetti@clinicaleterrazze.com

Key words: Cardiac resynchronization therapy; exercise; rehabilitation.

Received for publication: 11 November 2016

Accepted for publication: 20 September 2017

(C) Copyright M. Ambrosetti et al., 2017

Tipografia PI-ME Editrice, Italy

Monaldi Archives for Chest Disease 2017; 87:791

doi: 10.4081/monaldi.2017.791

This article is distributed under the terms of the Creative Commons Attribution Noncommercial License (by-nc 4.0) which permits any noncommercial use, distribution, and reproduction in any medium, provided the original author(s) and source are credited.

\section{Cardiac resynchronization therapy in heart failure}

Heart failure (HF) is a clinical syndrome characterized by typical symptoms (e.g., breathlessness, ankle swelling and fatigue) leading to marked disability and impaired life expectancy. The prevalence of HF is approximately $1-2 \%$ of the adult population in developed countries, rising to $\geq 10 \%$ among people $>70$ years of age [1-3]. Cardiac resynchronization therapy (CRT) represents an important advancement in HF treatment by targeting ventricular dyssynchrony, a condition occurring in up to a third of patients with severe systolic HF [4]. CRT is based on the addiction of a third left ventricular pacing lead to a standard pacemaker (CRT-P) or defibrillator system (CRT-D) that generally includes only two right side leads (Figure 1) The left side lead is generally placed into the lateral or posterolateral wall of the left ventricle, and when the two leads are activated, a coordinated pacing of the left ventricle and right ventricle is obtained [5]. Several randomized, controlled clinical studies have demonstrated that CRT has several positive clinical effects, including: improvement in cardiac function and structure, quality of life and exercise capacity, as well as reduction in heart failure hospitalization and mortality from progressive heart failure (Table 1). Moreover, by improving cardiac performance and efficiency, CRT favorably impacts cardiac autonomic control, potentially contributing to reverse remodeling of the left ventricle [6]. As in the case of implantable cardioverter defibrillator (ICD) devices [7], implantation policies may have significant impact on public health expenditure and appropriate patient selection for CRT is needed in order to maximize its benefits and cost-effectiveness. To date, European class //level A guidelines [8] recommend CRT for: i) symptomatic patients with HF in sinus rhythm with a QRS duration $\geq 150$ msec and LBBB QRS morphology and LVEF $\leq 35 \%$ despite optimal medical treatment in order to improve symptoms and reduce morbidity and mortality, and ii) patients with heart failure with reduced ejection fraction regardless of NYHA class, rather than right ventricular pacing, who have an indication for ventricular pacing because of high degree AV block, in order to reduce morbidity (patients with atrial fibrillation included).

\section{Cardiac resynchronization therapy in the cardiac rehabilitation setting}

Information about the impact of CRT in the Cardiac Rehabilitation (CR) setting, both in terms of characteristics of patients and program outcomes, is very scant. In the HF-action study [9], a controlled trial investigating outcomes of exercise training in 2331 outpatients with 
$\mathrm{HF}$ and left ventricular ejection fraction $\leq 35 \%$ randomized to usual care plus exercise training or usual care alone, $19 \%$ of patients had CRT. Patients with pacing devices were older and with lower functional capacity, as compared with those without devices.

Recently, the Cardiac Implantable Electronic Device in Cardiac Rehabilitation (CIED-CR) survey was carried out by the Italian Association of Cardiac Prevention and Rehabilitation (GICR-IACPR): data on cardiac device recipients were collected among different target populations entering CR programmes in 26 facilites, both in the inpatient and out-patient setting. Patients with CRT accounted for $28 \%$ of the HF group, with a CRT-D/CRT-P ratio of 7:1 (data presented at the 2016 of the GICR-IACPR Meeting). It was also estimated that near $5 \%$ of HF patients referred to CR in Italy may be candidates for CRT implantation.

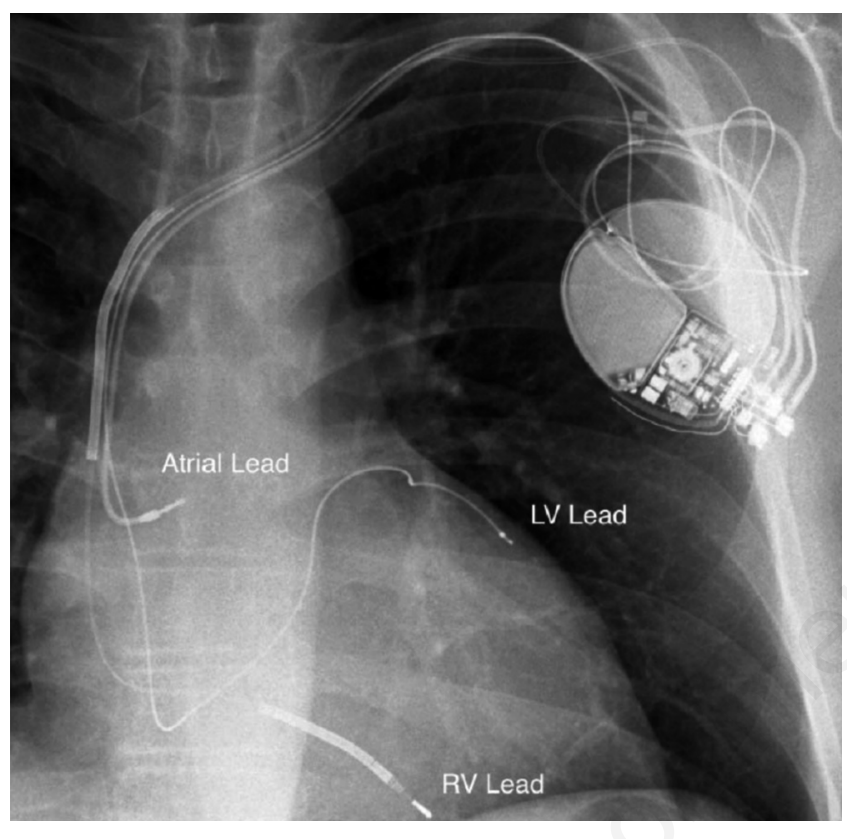

Figure 1. Position of pacing leads in cardiac resynchronization therapy aimed at restoring the mechanical sequence of ventricular activation and contraction.

\section{Effects of exercise in patients with cardiac resynchronization therapy}

As both exercise training (ET) and CRT improve exercise tolerance in HF patients, the combination of the two interventions may enhance the benefits. These improvements appear to be reasonably explained by both the enhancement of cardiac function induced by the device and by the improved peripheral (muscular and vascular) effects of exercise [10]. Exercise performance changes have been evaluated in terms of increased 6-minute walk test distance, increased treadmill exercise duration, increased $\mathrm{VO}_{2}$ peak, and increased anaerobic threshold among different studies, and generally were associated with improvements of hemodynamic status, quality of life, quality of sleep, depression and endothelial dysfunction [11-14].

Belardinelli et al. [12] determined the effects of a moderate exercise training program on functional outcomes in patients with chronic HF and NYHA class II-III (LVEF $31 \pm 7 \%$ ). Patients with ongoing exercise program were trained at an intensity approximating $60 \%$ of $\mathrm{VO}_{2}$ peak and, after 8 weeks, showed improvements in peak $\mathrm{VO}_{2}$, endotheliumdependent dilatation of the brachial artery, and quality of life. Conraads et al. [13] confirmed the cumulative effects of CRT plus exercise training in patients with $\mathrm{HF}$ and dyssynchrony by means of $\mathrm{VO}_{2}$ peak, maximal workload, and circulatory power, with improvements of more than two-fold as compared to CRT only. Patwala et al. [14] also documented further improvements in functional, exercise, hemodynamic, and quality of life measures in CRT patients after three months from implantation when exercise was added as a further intervention. In this study a significantly better skeletal muscle function depending on the frequency and duration of the exercise period was also shown.

Apart from functional capacity, the effects of combining CRT and exercise training on other major cardiovascular outcomes are not well known. Early physical activity, averaged over a 30-day window early after CRT implantation, has been recently found to predict death or HF hospitalization [15], but unfortunately no detailed information about the effect of structured ET on major cardiovascular events after CRT is available. The "Exercise Training Following Cardiac Resynchronization Therapy in Patients With Chronic Heart Failure" randomized study [16] is currently evaluating if adding an ET program following CRT may provide better clinical benefits than CRT alone. However, patients with chronic HF do not exhibit impaired exercise capacity only, but also

Table 1. Summary of evidence from major randomized clinical trials on cardiac resynchronization therapy. Adapted from: Sarzi Braga S, La Rovere MT, Pedretti RFE. Int J Cardiol 2006;09:118-20; with permission.

\begin{tabular}{|c|c|c|}
\hline Trial & Inclusion & Main findings \\
\hline \multicolumn{3}{|r|}{ CRT us OMT } \\
\hline MUSTIC & NYHA III, LVEF $<35 \%$, QRS $\geq 150$ & $\begin{array}{l}\text { CRT-P improved } 6 \mathrm{MWT} \text {, NYHA class, QoL, peak } \mathrm{VO}_{2} \text {, reduced } \mathrm{LV} \text { volumes and MR and reduced } \\
\text { hospitalizations }\end{array}$ \\
\hline MIRACLE & NYHA III-IV, LVEF $<35 \%$, QRS $\geq 130$ & Decrease in HF hospitalizations; improvement in 6MWT, LVEF, MR, quality of life, and NYHA class \\
\hline COMPANION & NYHA III-IV, LVEF $<35 \%$, QRS $\geq 120$ & CRT-P and CRT-D reduced all-cause mortality or hospitalization \\
\hline CARE-HF & NYHA III-IV, LVEF $<35 \%$, QRS $\geq 120$ & CRT-P reduced all-cause mortality and hospitalization and improved NYHA class and QoL \\
\hline \multicolumn{3}{|r|}{ CRT us ICD } \\
\hline MIRACLE-ICD & NYHA III-IV, LVEF $<35 \%$, QRS $\geq 130$ & CRT-D improved NYHA class, QoL, peak $\mathrm{VO}_{2}$ \\
\hline CONTAK-CD & NYHA II-IV, LVEF $<35 \%$, QRS $\geq 120$ & CRT-D improved 6MWT, NYHA class, QoL, reduced LV volume and increased LVEF \\
\hline MIRACLE-ICD II & NYHA II, LVEF $<35 \%$, QRS $\geq 120$ & CRT-D improved NYHA class, $\mathrm{VE} / \mathrm{CO}_{2}$ and reduced $\mathrm{LV}$ volumes and improved LVEF \\
\hline MADIT-CRT & NYHA I-II, LVEF $<30 \%$, QRS $\geq 130$ & CRT-D reduced HF hospitalizations or all-cause mortality and LVESV. \\
\hline RAFT & NYHA II-III, LVEF $<30 \%$, QRS $\geq 120$ & Decrease in death or hospitalization from $\mathrm{HF}$ \\
\hline
\end{tabular}


neurohormonal excitation, specifically increased resting sympathetic nerve activity, first measured by plasma norepinephrine levels. Neurohormonal activation is the principle mediator of the peripheral vasoconstriction characteristic of untrained HF patients, which may be also exacerbated by other non-neuronal factors, including blunted nitric oxide-mediated endothelial reactivity. In addition, HF patients may have diminished resting renal cortical blood, resulting in progressive activation of the renin angiotensin system. Finally, HF causes skeletal myopathy, with a shift from oxidative muscle fibers to glycolytic muscle fibers [17].

ET per se showed to reduce norepinephrine levels and central sympathetic nerve outflow, while enhancing vagal control and improving heart rate variability, in HF patients. The mechanisms underlying the reduction in the sympathetic modulation in exercise-trained HF patients remain uncertain, and changes in muscle mechanoreflex and metaboreflex sensitivity have been advocated [18]. ET also produces significant reduction in the local expression of cytokines and increase nitric oxide synthesis, with beneficial effects on endothelium [19]. On the other hand, CRT per se improves endothelial function [19] and - at least in responder patients - cardiac sympathetic nerve activity [20]. The hypothesis that ET could work synergistically with CRT to ameliorate pathophysiologic abnormalities in these patients is intriguing, but unfortunately not yet supported by strong evidence. Recently, studies using microneurography and evaluating $\mathrm{Ca} 2+$-handling gene expression at a muscle level have shown that including ET in the treatment of patients with HF, even following CRT, would further reduce muscle sympathetic nerve activity and vasoconstriction [21].

\section{How to prescribe exercise in patients with heart failure who have cardiac resynchronization therapy}

In the context of CR programmes, ET is delivered as part of a comprehensive and multidisciplinary approach, and core components of such intervention in HF patients with CRT have been well indentified by EACPR Policy Statement [22]. These include patient-risk assessment, physical activity counselling, ET, diet/nutritional counselling, weight control management, lipid management, blood pressure monitoring, smoking cessation, psychosocial management, and vocational management.
When prescribing exercise in CRT patients, wound evaluation both in terms of skin and heart muscle wire insertion has to be preliminary obtained, as far as complications during placement of device wires.

For CRT-D devices details of firing need to be provided, including ATP and shock thresholds, mode (VT or VF), rapid onset setting, and sustained arrhythmia period before device discharge. Information is essential in order to maintain exercise heart rates not exceeding ICD therapy thresholds, and ideally set between 10 to 20 beats below first line therapy thresholds. Exercise prescription should utilize one of the standard best-practice approaches of functional evaluation and monitoring, e.g. $\mathrm{VO}_{2}$, measured heart rate or rating of perceived exertion. Caution is required when prescribing exercise intensity based on estimated heart rate approaches, since the risk of targeting exercise heart rate above the detection threshold of the ICD: in this view, it is recommended that maximal heart rate needs to be measured rather than estimated in this patient population. Conversely, for monitoring purposes of exercise intensity, rating of perceived exertion may be the preferred method because the chronotropic response may be impaired in people with CRT [23]. Other possible limiting factors associated with pacing therapy that should be considered in the exercise prescription and monitoring are the following: i) in case of paced atrial rate (or set at a fixed rate) a blunted or delayed heart rate response to exercise may occur; and ii) ECG changes (ST-segment depression) associated with myocardial ischemia may not be visible, for which close clinical monitoring is required [24].

When prescribing exercise therapy in CHF patients undergoing CRT, it is also important to consider that about one third of patients are nonresponder to the device and may even show worsening symptoms after implantation, as far as device malfunction or infections in up to $5 \%$ of cases may occur [24]: in these situations appropriate diagnosis and modulation or interruption of the ET program is mandatory.

A proposal for ET prescription in CRT recipients in the setting of CR is summarized in Table 2. To date, there is no sufficient evidence regarding efficacy and safety of interval training in CRT, therefore aerobic training refers to endurance continuous exercise only. Further information about the use of aerobic interval training in CRT is expected by the ongoing "Exercise Training Following Cardiac Resynchronization Therapy in Patients With Chronic Heart Failure" randomized study [16]. Moreover, the most appropriate time window to start resistance training is not well defined, and therefore, this type of exercise is actually not recommended in the first weeks post-implant.

Table 2. Training modalities for cardiac resynchronization therapy patients in the Cardiac Rehabilitation setting.

\begin{tabular}{|c|c|c|c|}
\hline Endurance training & & Resistance training & \\
\hline Frequency & 3-5 day/week & Frequency & 2-3 day/week \\
\hline Modality of exercise & $\begin{array}{l}\text { Most exercises should be performed } \\
\text { standing, with horizontal and seated } \\
\text { arm exercises kept to a minimum. } \\
\text { Seated arm exercise to be avoided or } \\
\text { performed at a lower intensity }\end{array}$ & Modality of exercise & $\begin{array}{l}\text { All major muscle groups (the range of movement during } \\
\text { shoulder resistance training should take account of lead } \\
\text { issues; excessive shoulder flexion to be avoided) }\end{array}$ \\
\hline Intensity & $\begin{array}{l}\text { - } \text { Resting heart rate }+40-60 \% \\
\text { heart rate reserve } \\
\text { - Resting VO2 + 40-60\% VO2 reserve }\end{array}$ & Intensity & $\begin{array}{l}\text { 2-3 sets with 10-12 repetitions per set at 40-70\% 1-RM } \\
\text { with a full recovery period ( }>1 \text { min) between each set } \\
\text { If 1-RM evaluation not available, RPE of } 12-15 \\
\text { on the Borg 6-20 scale may be adopted }\end{array}$ \\
\hline Session duration & 30-40 min/day & --- & --- \\
\hline Warm-up and cool-down & 10 to 15 minutes & Warm-up and cool-down & 10 to 15 minutes \\
\hline Program duration & 12 weeks or more & Program duration & 12 weeks or more \\
\hline
\end{tabular}

RPE, rate of perceived exertion; 1-RM, one repetition maximum. 
Finally, tele-monitoring of CRT devices is of increasing importance in the long-term management of these patients, particularly for CR purposes, in order to modulate exercise prescription and promote adherence, and further evidence is needed on this topic [25].

\section{Conclusions}

In the modern era of CR, patients with $\mathrm{HF}$ and CRT represent an increasing target group for intervention, both with the aim to improve functional capacity and favourably affect prognosis. The prescription of ET, always tailored on patient needs and disabilities during CR programmes, needs to be furtherly individualized in HF patients with CRT, also considering device-related aspects. Nowadays, CRT patients engage most of the ET strategies offered by CR, and accumulation of observational research in this patient group may help to better define specific physical training programmes.

\section{References}

1. Mosterd A, Hoes AW. Clinical epidemiology of heart failure. Heart 2007; 93:1137-46.

2. Bleumink GS, Knetsch AM, Sturkenboom MCJM, et al. Quantifying the heart failure epidemic: prevalence, incidence rate, lifetime risk and prognosis of heart failure The Rotterdam Study. Eur Heart J 2004; 25:1614-9.

3. Ceia F, Fonseca C, Mota T, et al. Prevalence of chronic heart failure in Southwestern Europe: the EPICA study. Eur J Heart Fail 2002;4:531-9.

4. Cleland JG, Swedberg K, Follath F, et al. The EuroHeart Failure Survey programme - a survey on the quality of care among patients with heart failure in Europe. Part 1: Patient characteristics and diagnosis. Eur Heart J 2003;5:442-63.

5. Beshai JF, Khunnawat C, Lin AC. Mechanical dyssynchrony from the perspective of a cardiac electrophysiologist. Curr Opin Cardiol 2008;23:447-51.

6. Sarzi Braga S, La Rovere MT, Pedretti RFE. Baroreflex sensitivity normalization after cardiac resynchronization therapy. Int J Cardiol 2006; 09:118-20.

7. Pedretti RFE, Curnis A, Massa R, et al. Proportion of patients needing an implantable cardioverter defibrillator on the basis of current guidelines: impact on healthcare resources in Italy and the USA. Data from the ALPHA study registry. Europace 2010; 12:1105-11.

8. Ponikowski P, Voors AA, Anker SD, et al. 2016 ESC Guidelines for the diagnosis and treatment of acute and chronic heart failure. European Heart J 2016;37:2129-200.

9. Zeitler EP, Piccini JP, Hellkamp AS, et al. Exercise training and pacing status in patients with heart failure: results from HF-ACTION. J Card Fail 2015;21:60-7.

10. Iliou MC, Blanchard JC, Lamar-Tanguy A, et al. Cardiac rehabilitation in patients with pacemakers and implantable cardioverter defibrillators. Monaldi Arch Chest Dis 2016;86:756.

11. Iliou MC, Alonso C, Cristofini P, et al. Exercise training after cardiac resynchronization in chronic heart failure. Results of a pilot study. Eur J Cardiovasc Prev Rehab 2004;10:402.
12. Belardinelli R, Capestro F, Misiani A, et al. Moderate exercise training improves functional capacity, quality of life, and endotheliumdependent vasodilation in chronic heart failure patients with implantable cardioverter defibrillators and cardiac resynchronization therapy. Eur J Cardiovasc Prev Rehabil 2006;13:818-25.

13. Conraads V, Vanderheyden M, Paelinck B, et al. The effect of endurance training on exercise capacity following cardiac resynchronization therapy in chronic heart failure patients: a pilot trial. Eur J Cardiovasc Prev Rehabil 2007;14:99-106.

14. Patwala A, Woods P, Sharp L, et al. Maximizing patient benefit from cardiac resynchronization therapy with the addition of structured exercise training. J Am Coll Cardiol 2009;53:2332-9.

15. Conraads VM, Spruit MA, Braunschweig F, et al. Physical activity measured with implanted devices predicts patient outcome in chronic heart failure. Circ Heart Fail 2014;7:279-87.

16. Maria Helena Santa-Clara Pombo Rodrigues. Exercise training following cardiac resynchronization therapy in patients with chronic heart failure. [https:/clinicaltrials.gov/ct2/show/NCT02413151].

17. Negrao CE, Middlekauff HR, Gomes-Santos IL, Antunes-Correa LM. Effects of exercise training on neurovascular control and skeletal myopathy in systolic heart failure. Am J Physiol Heart Circ Physiol 2015;308:792-802.

18. Abreu A, Santa Clara H. Imaging predictive factors and exercise training in patients submitted to cardiac resynchronization. Monaldi Arch Chest Dis 2016;86:760.

19. Enomoto K, Yamabe H, Toyama K, et al. Improvement effect on endothelial function in patients with congestive heart failure treated with cardiac resynchronization therapy. J Cardiol 2011;58:69-73.

20. Martignani C, Diemberger I, Nanni C. Cardiac resynchronization therapy and cardiac sympathetic function. Eur J Clin Invest 2015; 45:792-9.

21. Nobre TS, Antunes-Correa LM, Groehs RV. Exercise training improves neurovascular control and calcium cycling gene expression in patients with heart failure with cardiac resynchronization therapy. Am J Physiol Heart Circ Physiol 2016;311:1180-8.

22. Piepoli MF, Corrà U, Adamopoulos S, et al. Secondary prevention in the clinical management of patients with cardiovascular diseases. Core components, standards and outcome measures for referral and delivery: a policy statement from the cardiac rehabilitation section of the European Association for Cardiovascular Prevention \& Rehabilitation. Endorsed by the Committee for Practice Guidelines of the European Society of Cardiology. Eur J Prev Cardiol 2014;21:664-81.

23. Haennel RG. Exercise rehabilitation for chronic heart failure patients with cardiac device implants. Cardiopulm Phys Ther J 2012;23:23-8.

24. McAlister FA, Ezekowitz J, Hooton N, et al. Cardiac resynchronization therapy for patients with left ventricular systolic dysfunction: a systematic review. JAMA 2007;297:2502-14.

25. Smolis-Bak E, Dabrowski R, Piotrowicz E, et al. Hospital-based and telemonitoring guided home-based training programs: effects on exercise tolerance and quality of life in patients with heart failure (NYHA class III) and cardiac resynchronization therapy. A randomized, prospective observation. Int J Cardiol 2015; 199:442-7. 